\title{
Efeitos do método pilates na funcionalidade e qualidade de vida na doença de Parkinson
}

The effects of the pilates method on functionality and quality of life in Parkinson's disease

\author{
Efectos del método pilates sobre la funcionalidad y la calidad de vida em la enfermedad \\ de Parkinson
}

Lydiane Kellen Benneterre de Araújo ${ }^{1}$, Leilane da Fonseca de Oliveira Silva ${ }^{1}$, Larissa Natsumi Hosoda Mineshita ${ }^{2}$, Marlyce Brasil Lopes ${ }^{2}$, Bento Edgar de Almeida Crisostomo ${ }^{1}$, Larissa Salgado de Oliveira Rocha ${ }^{3}$, Elysson Raul Ferreira Batista ${ }^{3}$, Ana Júlia Cunha Brito ${ }^{4}$, Mariana dos Anjos Furtado de Sá ${ }^{2}$, Dayse Danielle de Oliveira Silva ${ }^{2 *}$.

\section{RESUMO}

Objetivo: Avaliar os efeitos do Método Pilates sobre a funcionalidade e qualidade de vida (QV) em indivíduos com a Doença de Parkinson (DP). Métodos: Trata-se de um estudo piloto randomizado, do tipo duplo cego e controlado. A amostra inicial foi composta por 20 indivíduos com a DP, de ambos os sexos, com idade média de 64,5 \pm 7,2 anos, subdivididos em: Grupo Pilates (GP), submetidos a um protocolo baseado no método Pilates, e Grupo Controle (GC) submetidos a um protocolo de cinesioterapia, ambos trabalhavam os mesmos grupos musculares e realizaram duas sessões semanais, com duração de 45 minutos, totalizando 10 sessões. Os instrumentos utilizados foram o Unified Parkinson's Disease Scale (UPDRS), Questionário de Qualidade de vida PDQ-39, e a Escala de Hoehn \& Yahr (HY - Degree of Disability Scale). Resultados: Apenas 12 participantes concluíram o protocolo, $\mathrm{GP}=7$ e $\mathrm{GC}=5$. Foram observadas diferenças intragrupos nos aspectos clínicos gerais da DP (UPDRST) do GP $(p=0.046)$, sem interferir de forma significativa sobre a funcionalidade (UPDRSII e UPDRSIII) e a QV. Conclusão: O Método Pilates, comparado à cinesioterapia, apresentou melhores efeitos sobre os aspectos clínicos gerais da DP mas não influenciou a funcionalidade e a QV nos participantes analisados.

Palavras chave: Pilates, Doença de Parkinson, Qualidade de vida, Fisioterapia.

\begin{abstract}
Objective: To evaluate the effects of the Pilates Method on functionality and quality of life (QOL) in individuals with Parkinson's Disease (PD). Methods: This is a randomized, double-blind, controlled pilot study. The initial sample consisted of 20 individuals with PD, of both sexes, with an average age of $64.5+$ 7.2 years, subdivided into: Pilates Group (GP), submitted to a protocol based on the Pilates method, and Group Control (CG) submitted to a kinesiotherapy protocol, both worked the same muscle groups and performed two weekly sessions, lasting 45 minutes, totaling 10 sessions. The instruments used were the Unified Parkinson's Disease Scale (UPDRS), Quality of Life Questionnaire PDQ-39, and the Hoehn \& Yahr Scale (HY - Degree of Disability Scale). Results: Only 12 participants completed the protocol, GP = 7 and $\mathrm{GC}=5$. Intragroup differences were observed in the general clinical aspects of PD (UPDRST) of the GP $(p=$ 0.046), without significantly interfering with functionality (UPDRSII and UPDRSIII) and QOL. Conclusion: The Pilates Method, compared to kinesiotherapy, had better effects on the general clinical aspects of PD, but did not influence functionality and QOL in the analyzed participants.
\end{abstract}

Keywords: Pilates, Parkinson's disease, Quality of life, Physiotherapy.

1 Universidade da Amazônia (UNAMA), Belém - Pará.

2 Universidade Estadual do Pará (UEPA), Belém - Pará. *E-mail: daysesilva@uepa.br

${ }^{3}$ Centro Universitário do Estado do Pará (CESUPA), Belém - Pará.

${ }^{4}$ Faculdade Brasil Inteligente S/S (COSMOPOLITA), Belém - Pará.

SUBMETIDO EM: 2/2020

ACEITO EM: 3/2020

PUBLICADO EM: 5/2020

REAS/EJCH | Vol.12(6) | e3201 | DOI: https://doi.org/10.25248/reas.e3201.2020 Página 1 de 10 


\section{RESUMEN}

Objetivo: Evaluar los efectos del método Pilates sobre la funcionalidad y la calidad de vida (QOL) en personas con enfermedad de Parkinson (EP). Métodos: Este es un estudio piloto aleatorizado, doble ciego, controlado. La muestra inicial consistió en 20 individuos con EP, de ambos sexos, con una edad promedio de $64.5+7.2$ años, subdivididos en: Grupo Pilates (GP), sometidos a un protocolo basado en el método Pilates, y Grupo Control (CG) sometido a un protocolo de kinesioterapia, ambos trabajaron los mismos grupos musculares y realizaron dos sesiones semanales, con una duración de 45 minutos, con un total de 10 sesiones. Los instrumentos utilizados fueron la Escala Unificada de Enfermedad de Parkinson (UPDRS), el Cuestionario de Calidad de Vida PDQ-39 y la Escala Hoehn \& Yahr (Escala de Grado de Discapacidad HY). Resultados: Solo 12 participantes completaron el protocolo, GP $=7$ y GC $=5$. Se observaron diferencias intragrupales en los aspectos clínicos generales de la EP (UPDRST) de la GP $(p=0.046)$, sin interferir significativamente con la funcionalidad (UPDRSII y UPDRSIII) y QOL. Conclusión: El método Pilates, en comparación con la kinesioterapia, tuvo mejores efectos en los aspectos clínicos generales de la $E P$, pero no influyó en la funcionalidad y la calidad de vida en los participantes analizados.

Palabras clave: Pilates, Enfermedad de Parkinson, Calidad de vida, Fisioterapia.

\section{INTRODUÇÃO}

A Doença de Parkinson (DP) é considerada como uma afecção neurológica crônica que afeta o Sistema Nervoso Central (SNC) (MELLO MPB e BOTELHO ACG, 2010) descrita como a segunda doença neurodegenerativa mais comum, atingindo aproximadamente seis milhões de pessoas ao redor do mundo, cerca de $2 \%$ da população com idade superior a 60 anos (BOSE A e BEAL MF, 2016; ROEDER L, et al.; 2015). No Brasil estima-se uma prevalência em torno de 0,7\% em pessoas com idade entre 60 e 69 anos, e de 1,5\%, para indivíduos entre 70 e 79 anos (AYRES A, et al., 2017).

As consequências ocasionadas pela DP acarretam importantes déficits motores e cognitivos nos indivíduos acometidos, notáveis alterações na marcha e equilíbrio, com desenvolvimento de incapacidade de realizar tarefas essenciais básicas devido à lentidão e dificuldade de iniciar o movimento de forma voluntária (KING LA e HORAK FB, 2009; MACLEOD AD, et al., 2014)

Tais efeitos deletérios contribuem com modificações significativas na capacidade cognitiva e biopsicossocial dos indivíduos afetados, ocasionando consequências sobre a funcionalidade e a qualidade de vida (QV) (NAVARRO-PETERNELLA FM e MARCON SS, 2012). Fatores estes, que justificam a necessidade de protocolos específicos de tratamento voltados a minimizar e retardar os danos ocasionados pela DP a longo prazo.

Diante disso, recentes estudos ressaltam os benefícios induzidos por meio de programas de exercícios regulares que tendem a provocar mudanças no quadro geral dos pacientes, propiciando melhorias no estado de saúde, QV e funcionalidade (LAUZÉ M, et al., 2016; LIM HS, et al., 2016).

O método desenvolvido por Joseph Pilates (Método Pilates) pode influenciar positivamente na QV e funcionalidade de indivíduos com doenças neuromotoras, podendo ser aplicado na reabilitação de deficiências na marcha, equilíbrio, força e postura (SOYSAL TM, et al., 2016; KALRON A, et al., 2016) e consiste na utilização de princípios fundamentais que integram a concentração, controle, centralização, fluidez do movimento, precisão e respiração, empregados durante a execução dos exercícios com o objetivo de promover alongamento, relaxamento e ganho de equilíbrio, favorecendo o fortalecimento e aumento do tônus muscular, minimizando os desequilíbrios ocasionados entre músculos agonistas e antagonistas (BARKER AL, et al., 2015; KÜÇÜK F, et al., 2016).

Atualmente, o uso do método tornou-se bastante popular, sendo descrito por Küçük F, et al. (2016) como um modelo de exercício capaz de proporcionar múltiplos benefícios ao organismo, considerado como um modelo diferenciado de exercício por incorporar o componente respiratório em comparação a outras formas de treinamento. Todavia, relatos acerca da eficácia do método em doenças neurológicas eram pouco documentadas (SOYSAL TM, et al., 2016). 
Desta forma, é possível observar o crescimento e disseminação da aplicação do Método Pilates em pesquisas contemporâneas, com destaque para a Esclerose Múltipla (FOX EEH, et al., 2016) Fibromialgia (ALTAN L, et al., 2009) e em pacientes na fase crônica de Acidente vascular encefálico (AVE) (LIM HS, et al., 2016), no entanto, até o presente momento são escassos trabalhos voltados especificamente ao seu uso clínico na DP, justificando o objetivo principal deste trabalho, que consiste em avaliar os efeitos do Método Pilates sobre a funcionalidade e QV em indivíduos diagnosticados com a DP.

\section{MÉTODOS}

Trata- se de um estudo piloto randomizado, do tipo duplo cego e controlado, realizado com um protocolo adaptado do Método Pilates, baseado em publicações anteriores, nas disfunções clínicas da DP. O estudo foi realizado no Instituto Corpore Clínica-Escola, durante o período de janeiro a maio de 2017, após aprovação do Comitê de Ética em Pesquisa da Universidade da Amazônia - UNAMA, sob o número de parecer 1.872.327 e assinatura dos participantes do Termo de consentimento Livre e Esclarecido (TCLE).

A amostra foi composta inicialmente por 20 indivíduos, com diagnóstico clínico da DP, de ambos os sexos e idade média de 64,5 anos. Destes, apenas 19 foram incluídos na pesquisa por estarem de acordo com os critérios de inclusão. No decorrer da pesquisa, foi contabilizada uma perda amostral de sete participantes, sendo quatro correspondentes ao Grupo Pilates (GP), e três ao Grupo Controle (GC), totalizando ao término do estudo, 12 participantes, divididos em 7 participantes no GP e 5 no GC (Figura 1).

Figura 1 - Fluxograma de seleção dos participantes e aplicação do protocolo de pesquisa.

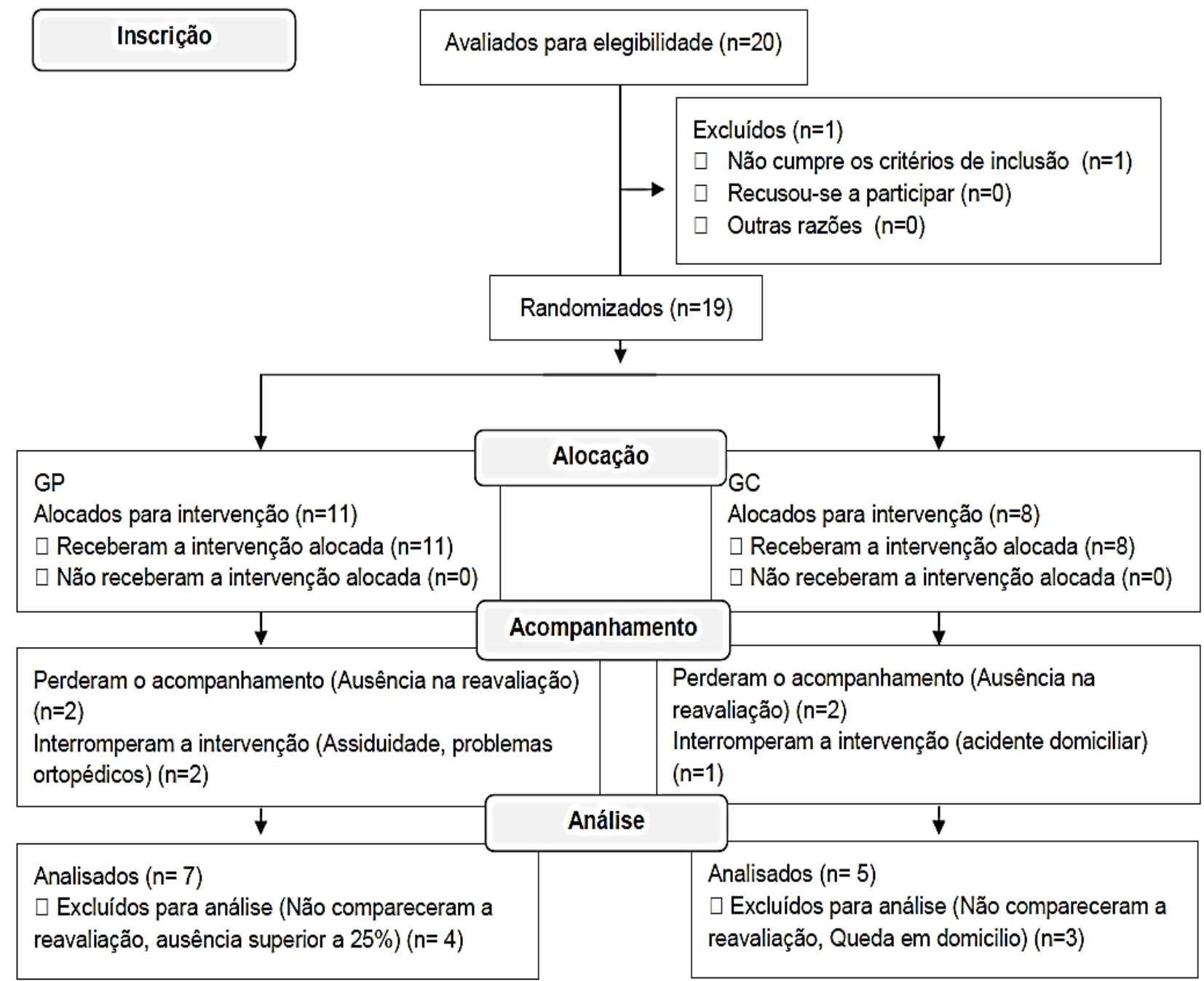

Fonte: Araújo LKB, et al., 2020. 
Foram incluídos neste estudo, indivíduos diagnosticados com a DP, não praticantes de atividade física, hemodinamicamente estáveis segundo as Diretrizes da Sociedade Brasileira de cardiologia (2016) que é de $\leq 120 / 80 \mathrm{mmHg}$ para indivíduos adultos, com o grau de estadiamento entre 1 e 3 da escala Hohen \& Yahr que estivessem na fase on da medicação durante o atendimento e que obtivessem uma pontuação $>26$ no teste Mini exame do estado mental (MEEM) considerando o ponto de corte de 19/20 para indivíduos não alfabetizados e de 23/24 para alfabetizados (HOEHN MM e YAHR MD, 1967, BERTOLUCCI PHF, et al., 1994, ALMEIDA OP, 1998).

Foram excluídos indivíduos que apresentassem quaisquer contraindicações ou comorbidades associadas que impedissem ou dificultassem a prática de exercícios físicos,clinicamente diagnosticados com outra doença neurológica associada à DP; indivíduos que apresentassem dificuldade de compreensão sobre o objetivo proposto ou incapacidade de atender ao comando verbal; com algum problema ortopédico debilitante ou que tivessem passado por procedimento cirúrgico nos últimos meses, participantes que se ausentassem por duas sessões consecutivas ou $25 \%$ do total das sessões.

Os participantes foram avaliados, antes e após a intervenção, por dois autores pesquisadores que desconheciam o grupo ao qual pertenciam, por meio dos instrumentos: Escala de funcionalidade Unified Parkinson`s Disease Scale (UPDRS) e Questionário de Qualidade de vida para Doença de Parkinson (PDQ39).

O UPDRS se baseia na avaliação e observação das principais manifestações clínicas da DP através do relato do paciente, contém 42 itens, subdivididos em quatro seções: no domínio I: avalia o estado mental, emocional e comportamental; II: correspondente à atividades da vida diária (AVD's); III: exame motor e IV complicações da terapia na semana que passou (GOETZ CG, et al., 2008).

O Questionário de Qualidade de vida PDQ-39, é considerado um instrumento altamente sensível e confiável para identificar os níveis de depleção na QV associada à DP, cuja pontuação varia de 0 a 100 , onde zero indica ausência de dificuldade ou problema, considerando que quanto menor o escore total, maior a QV do paciente avaliado (SILVA JAMG, et al., 2011). Além da Escala de Hoehn \& Yahr (HY- Degree of Disability Scale) utilizada para verificar o grau de estadiamento da DP relacionada aos sinais e sintomas apresentados, que avalia os níveis de incapacidade e estado geral dos indivíduos acometidos com a DP.

Após a avaliação, os participantes foram randomizados por meio de envelopes opacos, lacrados, entregues pelos pesquisadores avaliadores que não tinham conhecimento sobre a intervenção a ser realizada. Desta forma, os participantes foram distribuídos aleatoriamente em dois grupos: Grupo dos que foram submetidos ao Método Pilates (GP) e dos submetidos à cinesioterapia: o Grupo Controle (GC). Os participantes também desconheciam o grupo para o qual haviam sido designados.

Tanto o GP quanto GC realizaram duas sessões semanais com um período de intervenção de 45 minutos, totalizando 10 sessões, realizadas individualmente, com um protocolo de tratamento baseado em exercícios de fortalecimento, alongamentos utilizados na Cinesioterapia, voltados aos mesmos grupos musculares trabalhados no GP. Ambos os grupos foram atendidos em um estúdio de Pilates.

Em ambos os protocolos, inicialmente eram realizados os alongamentos associados à mobilidade de coluna, seguido dos exercícios de fortalecimento, estes foram introduzidos de acordo com o nível de classificação e dificuldade correspondente ao número progressivo de sessões, finalizando com o relaxamento, a dificuldade dos exercícios foi aumentando progressivamente por meio do incremento de molas, no método Pilates, e acessórios que permitissem uma maior resistência, instabilidade e controle postural, garantindo aos participantes a possibilidade de evoluir do nível básico para o intermediário, em cinco sessões correspondentes a cada nível, totalizando 10 sessões realizadas individualmente.

Todos os participantes foram orientados acerca da respiração correta durante o momento de esforço nos exercícios, bem como a evolução do treinamento com aumento constante do grau de complexidade dos exercícios. Foram respeitados os limites de cada paciente de acordo com o grau de dificuldade necessário para a mudança de nível. 
O protocolo do GP, adaptado do estudo de Küçük F, et al. (2016) para os participantes com DP, assim como os exercícios realizados no GC, elaborado por um pesquisador educador físico e uma fisioterapeuta, priorizaram os mesmos grupos musculares do GP e encontram-se na Quadro 1.

Quadro 1 - Comparação do programa de exercícios de acordo com os principais grupos musculares e nível de dificuldade a serem trabalhados nos GP e GC.

\begin{tabular}{|l|l|l|l|}
\hline Dificuldade & Pilates & Grupos musculares & Cinesioterapia \\
\hline Básico & $\begin{array}{l}\text { One Leg } \\
\text { Stretch }\end{array}$ & $\begin{array}{l}\text { Abdominais, flexores de quadril } \\
\text { e extensores de Joelho. }\end{array}$ & $\begin{array}{l}\text { Flexão de tronco e alongamento de } \\
\text { Tríceps Sural. }\end{array}$ \\
\hline Básico & $\begin{array}{l}\text { The Side } \\
\text { Kick }\end{array}$ & $\begin{array}{l}\text { Adutores e abdutores, flexores } \\
\text { e extensores de quadril. }\end{array}$ & $\begin{array}{l}\text { Flexo-extensão de quadril com } \\
\text { resistência,Adução e abdução de quadril } \\
\text { com uso de faixa elástica. }\end{array}$ \\
\hline Básico & Swimming & $\begin{array}{l}\text { Paravertebrais, glúteos e } \\
\text { subescapulares. }\end{array}$ & $\begin{array}{l}\text { Avanço de Ml com flexão de ombro com } \\
\text { bastão. }\end{array}$ \\
\hline Intermediário & $\begin{array}{l}\text { The } \\
\text { Hundreds }\end{array}$ & Abdominais. & $\begin{array}{l}\text { Flexão de tronco em DD com uso de bola } \\
\text { suíça. }\end{array}$ \\
\hline Intermediário & $\begin{array}{l}\text { Louble } \\
\text { Stretch }\end{array}$ & $\begin{array}{l}\text { Abdominais, flexores e } \\
\text { extensores de quadril, ombro e e } \\
\text { cotovelo. }\end{array}$ & $\begin{array}{l}\text { Exercício em ponte de ombros em DD, } \\
\text { extensão e flexão de MS com bastão. }\end{array}$ \\
\hline Intermediário & Swans & $\begin{array}{l}\text { Escapulares, adutores e } \\
\text { abdutores de ombro, e } \\
\text { extensores de cotovelo. }\end{array}$ & $\begin{array}{l}\text { Dissociação de cinturas de MS e MI com } \\
\text { uso de bastão. }\end{array}$ \\
\hline Intermediário & $\begin{array}{l}\text { The Onensores da coluna e e } \\
\text { Leg Kick }\end{array}$ & $\begin{array}{l}\text { Extensores de tronco; flexo- } \\
\text { extensores de ombro, quadril e } \\
\text { joelho. }\end{array}$ & $\begin{array}{l}\text { Fortalecimento de MS com alternância } \\
\text { para flexão e extensãode joelho e MI } \\
\text { com faixa elástica. }\end{array}$ \\
\hline
\end{tabular}

Legenda: MS- Membro superior; MI- Membro inferior, DD- Decúbito dorsal.

Fonte: Araújo LKB, et al., 2020, adaptado de Kuçuk F, et al., 2016.

Após o término das 10 sessões, os pacientes foram encaminhados aos pesquisadores avaliadores, e estes, sem o conhecimento de qual tratamento foram submetidos, realizaram a reavaliação dos participantes obedecendo os mesmos critérios da avaliação inicial.

A análise estatística foi feita por dois autores que desconheciam qual numeração havia sido atribuída ao GP e ao GC, apenas a pesquisadora responsável conhecia esse dado. Os dados foram analisados por meio do programa SPSS versão 20.0 .

Utilizou-se testes não paramétricos devido ao baixo $\mathrm{n}$ amostral, seguido pelo teste de Wilcoxon para análise das variáveis dependentes e quando independentes foram tratadas pelo teste de Mann-Whitney, além do método de Cohen Pooled utilizado para o cálculo do efeito do tratamento.

\section{RESULTADOS}

Dos 20 participantes recrutados, após avaliação inicial, 1 indivíduo foi excluído por apresentar $\mathrm{H} \& Y>3$, no GP 2 foram excluídos por problemas ortopédicos e 2 não compareceram à reavaliação, no GC 1 foi excluído por acidente domiciliar e 2 não compareceram à reavaliação, portanto apenas 12 concluíram o protocolo, sendo 7 no GP e 5 no GC, não sendo observadas diferenças antropométricas significantes entre os grupos (Tabela 1). 
Tabela 1 - Idade e características antropométricas dos grupos GP e GC.

\begin{tabular}{lccc} 
Variáveis & $\begin{array}{c}\text { GP }(\mathbf{n}=7) \\
\text { Mediana (Intervalo } \\
\text { interquartílico) }\end{array}$ & $\begin{array}{c}\text { Mediana (Intervalo } \\
\text { interquartílico) }\end{array}$ & p-valor \\
\hline Idade (anos) & $68(59-71)$ & $66(55.5-69.5)$ & 0.53 \\
\hline Peso (Kg) & $61(53-70)$ & $72(67-81)$ & 0.10 \\
\hline Altura & $155(147-163)$ & $164(160.5-171.5)$ & 0.07 \\
\hline
\end{tabular}

Legenda: $\mathrm{GP}=$ Grupo Pilates; $\mathrm{GC}=$ Grupo Controle.

Fonte: Araújo LKB, et al., 2020.

Os participantes submetidos ao protocolo baseado no método Pilates apresentaram melhora dos aspectos clínicos gerais da doença observados por meio da diminuição da pontuação total (UPDRST), no entanto as diferenças observadas nas subescalas (UPDRS I-IV) não permitiu afirmar qual área teria apresentado maior impacto nessa melhora (Tabela 2).

Tabela 2 - Análise intra grupos pós intervenção pelo método Pilates (GP) e pela Cinesioterapia (GP) sobre os aspectos clínicos e qualidade de vida na DP.

\begin{tabular}{|c|c|c|c|}
\hline Variáveis & \multicolumn{3}{|c|}{ GP $(n=7)$} \\
\hline & $\begin{array}{c}\text { Pré } \\
\text { Mediana (Intervalo interquartílico) }\end{array}$ & $\begin{array}{c}\text { Pós } \\
\text { Mediana (Intervalo interquartílico) }\end{array}$ & $p$-valor \\
\hline UPDRS I & $2(1-4)$ & $2(0-5)$ & 0.596 \\
\hline UPDRS II & $7(5-24)$ & $7(4-19)$ & 0.399 \\
\hline UPDRS III & $7(5-16)$ & $7(5-12)$ & 0.092 \\
\hline UPDRS IV & $4(4-5)$ & $5(2-7)$ & 0.931 \\
\hline UPDRS T & $20(16-54)$ & $17(14-53)$ & $0.046^{*}$ \\
\hline PDQ-39 & $19(16-54)$ & $21(15-53)$ & 0.173 \\
\hline Variáveis & \multicolumn{3}{|c|}{$G C(n=5)$} \\
\hline & $\begin{array}{c}\text { Pré } \\
\text { Mediana (Intervalo interquartílico) }\end{array}$ & $\begin{array}{c}\text { Pós } \\
\text { Mediana (Intervalo interquartílico) }\end{array}$ & $p$-valor \\
\hline UPDRS I & $0(0-5)$ & $1(0-2)$ & 0.285 \\
\hline UPDRS II & $14(2-18)$ & $12(1.5-16.5)$ & 0.063 \\
\hline UPDRS III & $15(5-16.5)$ & $12(4.5-13.5)$ & 0.279 \\
\hline UPDRS IV & $4(3.5-9.5)$ & $4(1-9.5)$ & 0.450 \\
\hline UPDRS T & $33(13,5-46)$ & $29(10.50-38)$ & 0.066 \\
\hline PDQ-39 & $28(14.5-47.5)$ & $24(6.5-29)$ & 0.225 \\
\hline
\end{tabular}

Legenda: $\mathrm{DP}=$ Doença de Parkinson; $\mathrm{GP}=$ Grupo Pilates; Grupo Controle=GC; Unified Parkinson`s Disease Scale =UPDRS; Questionário de Qualidade de vida para Doença de Parkinson = PDQ-39;" $>>0.05$.

Fonte: Araújo LKB, et al., 2020.

Quando comparados, não se observou, entre os protocolos aplicados, diferença sobre os aspectos clínicos e a qualidade de vida na DP (Tabela 3).

Tabela 3 - Comparação intergrupos do desempenho pré e pós intervenção nos aspectos clínicos e qualidade de vida na DP.

\begin{tabular}{lllllll}
\hline \multirow{2}{*}{ Variáveis } & \multicolumn{7}{c}{ Pós tratamento } \\
\cline { 2 - 7 } & GP & GC & \multicolumn{6}{c}{ GP } & GC & \\
\cline { 2 - 7 } & $(\mathrm{Ri})^{*}$ & $(\mathrm{Ri})^{*}$ & $\mathbf{p}$-valor & $(\mathrm{Ri})^{*}$ & $(\mathrm{Ri})^{*}$ & p-valor \\
\hline UPDRS I & 50.5 & 27.5 & 0.208 & 51.5 & 26.5 & 0.164 \\
\hline UPDRS II & 48 & 30 & 0.342 & 48 & 30 & 0.342 \\
\hline UPDRS III & 44 & 34 & 0.403 & 40.5 & 37.5 & 0.208 \\
\hline UPDRS IV & 50 & 28 & 0.232 & 46.5 & 31.5 & 0.435 \\
\hline UPDRS T & 44.5 & 33.5 & 0.435 & 43.5 & 34.5 & 0.372 \\
\hline PDQ39 & 45 & 33 & 0.467 & 49 & 29 & 0.284 \\
\hline
\end{tabular}

Legenda: $\mathrm{DP}=$ Doença de Parkinson; GP=Grupo Pilates; Grupo Controle=GC; Unified Parkinson`s Disease Scale=UPDRS; Questionário de Qualidade de vida para Doença de Parkinson=PDQ-39; *Soma dos postos (Ri). Fonte: Araújo LKB, et al., 2020. 
É importante avaliar o significado (a importância prática) dos resultados e eventuais diferenças encontradas entre as variâncias encontradas. A subescala I da UPDRS e a qualidade de vida (PDQ39) apresentaram um tamanho de efeito médio pós intervenção para o CG como exemplificado na Tabela 4.

Tabela 4 - Tamanho do Efeito do tratamento nas variáveis funcionalidade e qualidade de vida entre os períodos de análise, nos grupos Controle (GC) e Pilates (GP).

\begin{tabular}{lllll}
\hline \multirow{2}{*}{ Variáveis } & Pré tratamento & \multicolumn{3}{c}{ Póstratamento } \\
\cline { 2 - 5 } & Cohen's d & efeito-tamanho & Cohen's d & efeito-tamanho \\
\hline UPDRS I & 0.3397 & 0.1674 & 0.7861 & 0.3658 \\
\hline UPDRS II & 0.0537 & 0.0268 & 0.0882 & 0.0440 \\
\hline UPDRS III & -0.0408 & -0.0204 & -0.1275 & -0.0636 \\
\hline UPDRS IV & -0.3066 & -0.1515 & 0 & 0 \\
\hline UPDRS T & 0.0015 & 0.0007 & 0.0799 & 0.0399 \\
\hline PDQ39 & 0.3121 & 0.1542 & 0.6767 & 0.3205 \\
\hline
\end{tabular}

Fonte: Araújo LKB, et al., 2020.

\section{DISCUSSÃO}

No presente estudo, foram avaliados os efeitos de duas intervenções terapêuticas sobre os aspectos clínicos e qualidade de vida de 12 participantes com DP. Os resultados demostraram que não foi possível observar diferenças significativas intragrupos. Os participantes submetidos ao Método Pilates apresentaram melhora nos aspectos clínicos da DP.

Estes achados não corroboram com o estudo de Fox EEH, et al. (2016) para pacientes com EM, onde não foi observado melhoras após 12 semanas de Pilates quando comparado ao grupo de relaxamento que teve o mesmo tempo de intervenção. Porém houve resultados significantes sobre as medidas de equilíbrio e mobilidade para os exercícios de fisioterapia padronizados.

Fox EEH, et al. (2016), Karoln A, et al. (2016) que utilizaram o Método Pilates e compararam com a cinesioterapia em pacientes diagnosticados com EM, demostraram que ambos os grupos obtiveram um aumento significativo na velocidade da caminhada e comprimento do passo, porém, não foram observadas grandes vantagens com relação ao uso do Método Pilates, uma vez que os resultados foram semelhantes. Por outro lado, no protocolo de atendimento utilizado por Lim HS, et al. (2016), os resultados indicaram evidências de que o método inferiu positivamente na melhora do equilíbrio estático e dinâmico em pacientes pós AVE.

Além destes, outros estudos também avaliaram a eficácia do método Pilates, dentre eles, o estudo utilizado como base para realização desta pesquisa, desenvolvido por Küçük F, et al. (2016) para indivíduos com EM, que evidenciou resultados significativos para o uso do Método Pilates no equilíbrio, desempenho e esforço, antes e depois do tratamento, com melhora na função cognitiva e QV em comparação aos exercícios convencionais. Entretanto, não foi possível observar os mesmos achados clínicos nesta pesquisa com relação a QV e funcionalidade, considerando que o número de sessões pode não ter sido suficiente para promover uma resposta satisfatória sobre a plasticidade cerebral com consequente ativação neuromuscular nos participantes do GP quando comparados ao GC.

Com relação a isso, Agosti V, et al. (2016) fez uso do Método de Reeducação Postural Global (RPG) com objetivo de alcançar maior estabilidade e controle dinâmico, e observou uma maior variabilidade no padrão de marcha na DP por meio de respostas efetivas sobre a manutenção dos níveis basais dos músculos agonistas e antagonistas, semelhante ao que pode ter ocorrido no GC, onde possivelmente a cinesioterapia foi capaz de proporcionar uma maior adaptação sobre o sinergismo neuromuscular e isso pode ter influenciado na maior adesão ao respectivo protocolo de tratamento. Na presente pesquisa, o grupo submetido ao protocolo cinesioterapêutico apresentou redução da pontuação nos instrumentos UPDRS e PDQ-39, no entanto, sem significância o que pode ser atribuído ao baixo $n$ amostral. 
Como uma possível justificativa para as diferenças apresentadas entre os grupos neste estudo, destacase também a influência que o estado emocional pode ter exercido sobre os centros de comando centrais, uma vez que a insegurança e a instabilidade postural aumentam os níveis de tensão muscular, resultando em uma maior rigidez articular que tende a comprometer o equilíbrio existente entre os estados de tensãorelaxamento que encontram-se ainda mais comprometidos em pacientes com a DP como demostrou Solopoval A, et al. (2014) que analisou 25 indivíduos com a DP em comparação a controles saudáveis e observou uma maior rigidez durante os movimentos passivos em todos os grupos musculares dos membros inferiores, exceto para os extensores do pé, concluindo que em comparação a população normal, a rigidez articular na DP prejudica a execução de movimentos funcionais participantes, interferindo diretamente na prática de atividades físicas.

Desta forma, destacamos que a rigidez ocasionada pelos elevados níveis de tensão, pode ter sido um fator limitante para a adaptação completa ao protocolo baseado no Método Pilates, uma vez que a prática do mesmo se apresenta como um novo desafio para os centros de planejamento e controle motor, o que pode ter comprometido a fluidez e o controle do movimento considerados fundamentos básicos do método, que podem ser adquiridos e aprimorados mediante o treino constante (BARKER AL, et al., 2015; LAUZÉ M, et al., 2016).

Além disso, Capato TTC, et al. (2015) destaca que estratégias compensatórias ocorrem em consequência das alterações no centro de gravidade devido a projeção anterior do corpo, acarretando desarranjos estruturais, sobrecargas e falhas nas estruturas responsáveis pela sustentação corporal, gerando fraqueza muscular e inabilidade motora frente a realização de exercícios funcionais, fator este que em conjunto com as demais particularidades existentes na DP corroboraram com a necessidade de adaptação do protocolo original para o GP, uma vez que os exercícios avançados não puderam ser incorporados em decorrência da dificuldade na execução de exercícios em DV, com relatos de desconforto acerca das mudanças de decúbito que provocaram episódios de hipotensão apresentado por dois participantes nas primeiras duas sessões do GP.

Isso também poderia ser justificado em virtude da discrepância entre as faixas etárias nas amostras analisadas no estudo de Küçük F, et al. (2016) onde a média foi de 47,2 (GP) e 49,2 (GC) anos e no presente estudo foi de 65,4 (GP) e 63,2 (GC), sendo esta a faixa etária mais acometida pela DP de acordo com a literatura ${ }^{5}$, o que corresponde a uma melhor representatividade da amostra estudada. No GC, apenas três participantes apresentaram dificuldades em exercícios que exigiam movimentos coordenados de ambos os membros, dentre eles, apenas um não demonstrou evolução. Ademais, não foram relatados maiores desconfortos ou efeitos colaterais expressivos em nenhum dos grupos analisados. Conradsson D, et al. (2014) destacou efeitos colaterais semelhantes ao apresentado no GP após o uso de treinamento altamente desafiador e progressivo durante 12 semanas em pacientes idosos com a DP que resultaram em um episódio de tontura e uma queda, ratificando a hipótese acerca da repercussão que grandes modificações posturais exercem sobre a capacidade de execução de tarefas induzidas por meio da atividade física desafiadora na DP, os autores discorrem que o treino progressivo é viável para possibilitar a evolução clinica durante o período de treinamento.

Recentemente uma meta-analise feita por Suares-Iglesias D (2019), evidenciou que a eficácia do Pilates na DP demonstrou-se superior a outras formas de treinamento para melhorar a função de membros inferiores, destacando que o mesmo pode ser bastante desafiador em alguns casos, dentre os achados houve apenas um relato isolado de tontura leve, fadiga e câimbras, não sendo observados intercorrências na maioria dos ensaios analisados, os autores admitem que amostras maiores e protocolos randomizados com alta qualidade metodológica seriam necessários para comprovar de fato os benefícios do método, além disso, a média entre os programas de treino variou entre 2-3 sessões semanais de 60 minutos, durante 12 semanas, concordando com a presente pesquisa.

No entanto, pressupomos que um período de 10 sessões pode não ter sido suficiente para proporcionar uma adaptação efetiva com relação aos princípios do método junto ao acionamento dos músculos estabilizadores profundos (CORE), que podem não ter sido ativados ou recrutados corretamente, 
comprometendo as implicações posturais indispensáveis para se alcançar os benefícios esperados, considerando que para o criador do Método Joseph Pilates (1883-1967), um período de 20 sessões seria o número ideal para se observar os resultados no próprio corpo, 10 seria o tempo mínimo para se sentir os efeitos, e 30 a quantidade necessária para se alcançar um resultado ideal.

Também é valido destacar a influência de outros fatores como o tamanho da amostra e o período de intervenção em comparação aos demais estudos analisados, neste o $\mathrm{n}$ amostral foi de 12 participantes, com duração de 45 minutos, números inferiores aos descritos por Küçük $F$, et al. (2016) que dispunham de uma amostra final de 20 participantes, com 60 minutos de intervenção. No estudo de Lim HS, et al. (2016), 30 indivíduos receberam tratamento e 45 no estudo de Karoln A, et al. (2016) que não descreveu o tempo de intervenção.

Acreditamos que um número maior de sessões, maior tempo de intervenção aliado a aplicação de um protocolo mais específico para a DP, poderiam melhorar os resultados. Reiterando que a insipiência de estudos mais abrangentes, com programas de exercícios padronizados seriam substanciais para melhores comparações acerca dos resultados em pesquisas futuras.

Propondo que no tocante a melhora na QV para o GC, estima-se que a manutenção desta, deve ser medida primordial no tratamento de indivíduos portadores de doenças crônicas progressivas independentemente do método empregado, uma vez que o avanço dos sintomas motores provocam depleção dos níveis de QV com repercussões clínicas negativas, como concluíram Navarro-Peternella FM e Marcon SS (2012) ressaltando que a gravidade da doença apresenta tendência a piora da percepção na QV, nos aspectos funcionais e cognitivos.

Desta forma, os resultados sugerem que com relação a análise das médias pré e pós intervenção, verificou-se um maior efeito positivo da cinesioterapia sobre o a QV, obtendo-se um tamanho médio do efeito do tratamento para o GC, demonstrando uma possível melhora no estado emocional e psicológica dos participantes, destacando que embora tenham sido observados melhores resultados no GP, estes não foram significantes em comparação ao GC.

\section{CONCLUSÃO}

O presente estudo objetivou avaliar os efeitos de duas intervenções terapêuticas sobre os aspectos clínicos e qualidade de vida em indivíduos com DP. Entre os participantes da pesquisa, não foi possível observar diferenças intragrupos, os que foram submetidos ao Método Pilates e os que foram submetidos à cinesioterapia, no entanto o Método Pilates, comparado à cinesioterapia, apresentou melhores efeitos sobre os aspectos clínicos gerais da DP, por outro lado nenhum dos protocolos utilizados influenciou a funcionalidade e a QV dos participantes analisados, o que poderia ser atribuído a variáveis como o número de sessões ou o baixo $\mathrm{n}$ amostral, devendo ser objeto de novas pesquisas.

\section{AGRADECIMENTOS}

Nossos agradecimentos à Clínica-Escola pela disponibilidade do espaço de atendimento, pelo engajamento e colaboração de toda equipe para realização da pesquisa.

\section{REFERÊNCIAS}

1. AGOSTI V, et al. Erratum to: Effects of Global Postural Reeducation on gait kinematics in parkinsonian patients: a pilot randomized three-dimensional motion analysis study. Neurol Sci. 2016; 37(4): 523-524.

2. ALMEIDA OP. Mini exame do estado mental e o diagnóstico de demência no Brasil São Paulo. Arq. Neuro-Psiquiatr 1998; 56(3B): 605-612.

3. ALTAN L, et al. Effect of pilates training on people with fibromyalgia syndrome: a pilot study. Arch Phys Med Rehabil, 2009; 90(12): 1983-8.

4. AYRES A, JACINTO-SCUDEIRO LA, OLCHIK M. Instrumentos de avaliação clínica para disfagia orofaríngea na doença de Parkinson: revisão sistemática. Audiol Commun Res, 2017; 22: 1814. 
5. BARKER AL, et al. Feasibility of Pilates exercise to decrease falls risk: A pilot randomized controlled trial in community-dwellingolder people. ClinRehabil, Sep; 2015.

6. BERTOLUCCI PHF.et al. O mini-exame do mental em uma população geral: Impacto da escolaridade. Arquivos de Neuropsiquiatria, 1994; 52(1): 1-7.

7. BOSE A, BEAL MF. Mitochondrial dysfunction in Parkinson's disease. J Neurochem, 2016; 139: $216-231$.

8. CAPATO TTC, et al. Randomized controlled triad protocol: balance training with rhythmical cues to improve and maintain balance control in Parkinson's disease. BMC neurology, 2015; Sep (15): 162.

9. CONRADSSON D, et al. Is Highly Challenging and progressive balance training feasible in older adults with Parkinsons's Disease? Arch Physical Medicine and Rehabilitation, 2014; 95(5).

10. FOX EEH, et al. Effects of Pilates-Based Core Stability Training in Ambulant People with Multiple Sclerosis: Multicenter, Assessor-Blinded, Randomized Controlled Trial. Phys Ther. 2016; 96(8): 1170-8.

11. GOETZ CG, et al. Movement Disorder Society-Sponsored Revision of the Unified Parkinson's Disease Rating Scal (MDS-UPSRS): scale presentation and clinimetric testing results. Moviment Disorder, 2008; 23(15): 2129-2170.

12. HOEHN MM, YAHR MD. Parkinsonism: onset, progression and mortality. Neurology, 1967; 17(5): 427-42.

13. KALRON A, et al. Pilates exercise training vs. physical therapy for improving walking and balance in people with multiple sclerosis: A randomized controlled trial. ClinRehabil, Mar; 2016.

14. KING LA, HORAK FB. Delaying mobility disability in people with Parkinson disease using a sensori motor agility exercise program. PhysTher, 2009; 89(4): 384-393.

15. KÜÇÜK F, et al. Improvements in cognition, quality of life, and physical performance with clinical Pilates in multiple sclerosis: a randomized controlled trial. J Phys TherSci, 2016; 28(3): 761-8.

16. LAUZÉ, M, et al. The Effects of Physical Activity in Parkinson's Disease: A Review. Journal of Parkinson's Disease, 2016; 6(4): 685-698.

17. LIM HS, KIM YL, LEE SM. The effects of Pilates exercise training on tatic and dynamic balance in chronic stroke patients: a randomized controlled trial. Journal of Physical Therapy Science, 2016; 28(6): 1819-1824.

18. MACLEOD AD, et al. Mortality in Parkinson's disease: A systematic review and meta-analysis. Mov Disord, 2014; 29(13): 1615-1622.

19. MELLO MPB, BOTELHO ACG. Correlação das escalas de avaliação utilizadas na doença de Parkinson com aplicabilidade na fisioterapia. FisioterMov, 2010; 23(1): 121-127.

20. NAVARRO- PETERNELLA FM, MARCON SS. Qualidade de vida de indivíduos com Parkinson e sua relação com tempo de evolução e gravidade de doença; Rev. Latino -Am Enfermagem, 2012; 20(2).

21. ROEDER L, et al. Effects of Resistance Training on Measures of Muscular Strength in People with Parkinson's Disease: A Systematic Review and Meta-Analysis. PLoS ONE, 2015; 10(7).

22. SHEA S, MORIELLO G. Feasibility and outcomes of a classical Pilates program on lower extremity strength, posture, balance, gait, and quality off life in someone with impairments due to a stroke. J BodywMovTher, 2014; 18(3): 332-360.

23. SILVA JAMG, et al. Mensuração da qualidade de vida de indivíduos com a doença de Parkinson por meio do questionário PDQ-39. FisioterMov, 2011; 24(1): 41-6.

24. SOCIEDADE BRASILEIRA DE CARDIOLOGIA. 7aㅡ Diretriz Brasileira de Hipertensão Arterial. 2016. in: Arquivos brasileiros de cardiologia; 107(Supl. 3). Disponível em: www.arquivosonline.com.br. Acesso em 3 de setembro de 2016.

25. SOLOPOVA IA, et al. Investigation of muscle tone in patients with Parkinson's disease in unloading conditions. FiziolCheloveka_2014; 40(2): 14-21.

26. SOYSAL TM, et al. Effects of Pilates exercises on sensory interaction, postural controland fatigue in patients with multiple sclerosis. MultSclerRelatDisord, 2016; 7: 70-3.

27. SUARES-IGLESIAS D, et al. Benefits of Pilates in Parkinsons Disease: A Systematic Review an Meta-Analysis. Medicina (Kaunas), 2019; 55(8):476. 